with wound infection were more likely to have had recent exposure to salt water or shellfish.

Another multi-state US study ${ }^{6}$ identified ingestion of raw oysters as a risk factor for developing $V$. vulnificus septicaemia, but not for wound infection. Primary septicaemia occurred almost exclusively in people who had some underlying illness, particularly liver disease. Wound infection, on the other hand, resulted from exposure to seawater or freshly caught saltwater crabs, and appeared to affect people with no underlying illness. A case control study conducted in New Orleans ${ }^{3}$ found similar risk factors.

Liver disease, haemopoietic disorders, chronic renal insufficiency, haemochromatosis ${ }^{6}$, heavy alcohol intake and the use of immunosuppressants are risk factors for developing $V$. vulnificus septicaemia ${ }^{3}$. Other reported risk factors include thalassaemia ${ }^{4}$, diabetes, and leukaemia.

V. vulnificus wound infections may be mild and self limiting, or can progress to severe rapidly progressive cellulitis and myositis. The incubation period is short, with symptoms developing about 12 hours after contamination. The infection occurs in healthy as well as debilitated people 1 . Almost half (48\%) of cases in one study ${ }^{7}$ had an underlying disease such as diabetes, cirrhosis, gastric carcinoma and steroid-dependent asthma. While the presence of underlying disease does not appear to influence the risk of developing V. vulnificus wound infection, use of immunosuppressive drugs and having underlying diseases are associated with more severe outcomes for those who develop the condition.

V. vulnificus has also been reported in association with diarrhoea in patients reporting heavy alcohol intake, meningitis in a child with thalassaemia ${ }^{4}$, pulmonary infection, and endometriosis following undersea intercourse $^{1}$

\section{PREVENTION AND CONTROL}

Various recommendations have been made to reduce the risk of developing $V$. vulnificus infection, including:

Advising people with underlying illness to avoid shellfish injuries and contaminating any wounds with sea water during the warmer months ${ }^{1,7}$.

\section{INFLUENZA MONITORING}

here has been widespread community concern

that an influenza epidemic will spread within Australia this winter.

During the recent 1989-90 influenza epidemic [subtype A/England/427/88 (H3N2) - close to A/Shanghai/11/87 (H3N2)] in the United Kingdom, there was a reported peak number of some 800 deaths attributable to influenza ${ }^{1}$, the majority of these occurring in people over the age of 65 .

Worse influenza epidemics have occurred in the UK in the past. In 1969-70 an epidemic of A/Hong Kong/1/68 (H3N2) influenza occurred, with an estimated peak of 3,000 deaths per week, and in a 1975-76 A/Victoria/3/75 (H3N2) epidemic less than half as many influenza deaths were reported.

However, there are in fact important differences in the epidemiology of influenza between Australia and the UK. As can be seen from Fig 3, the UK had not experienced an epidemic of Influenza A (H3N2) since 1976. In contrast, Australians have been exposed to Influenza A (H3N2) virus in 1982, 1983, 1985 and 1989, making them less susceptible to these virus strains and decreasing the likelihood of a large influenza epidemic this year.

Should Australia experience an influenza epidemic in 1990 , initial cases could have occurred as early as May (as was the experience in the recent UK outbreak), or as late as July (as occurred in Australia during the moderate epidemic of 1989). The typical epidemic lasts five to nine weeks, peaking around the third to fifth week.

\section{Ongoing surveillance of viral isolates by}

Commonwealth Communicable Diseases Intelligence (CCDI) has revealed only 12 influenza isolates in the March and April periods - and only one was Influenza A subtype H3N2. It was noted that this was normal Influenza A activity for that particular time of year².

Compared with immunisation, natural acquisition of influenza appears to afford greater immunity against the disease and provides some protection against later emerging strains resulting from "antigenic drift'. This is the basis for not recommending influenza vaccine in otherwise healthy children and young adults.

To monitor the occurrence of influenza in the community, the Epidemiology and Health Services Evaluation Branch of the Department of Health and the Department of Community Medicine of the University of Sydney have established an influenza monitoring network. A total of 24 general practitioners throughout metropolitan Sydney will report on patients presenting with "'flu-like illness". These data will be collated with data from hospital separations and virology laboratories in order to identify the beginning, peak and end of an epidemic, if it does occur. 


\section{FIGURE 3}

DEATHS REPORTED WEEKLY IN

ENGLAND \& WALES (UPPER DIAGRAM),

AND NEW SOUTH WALES

(LOWER DIAGRAM) ${ }^{1}$
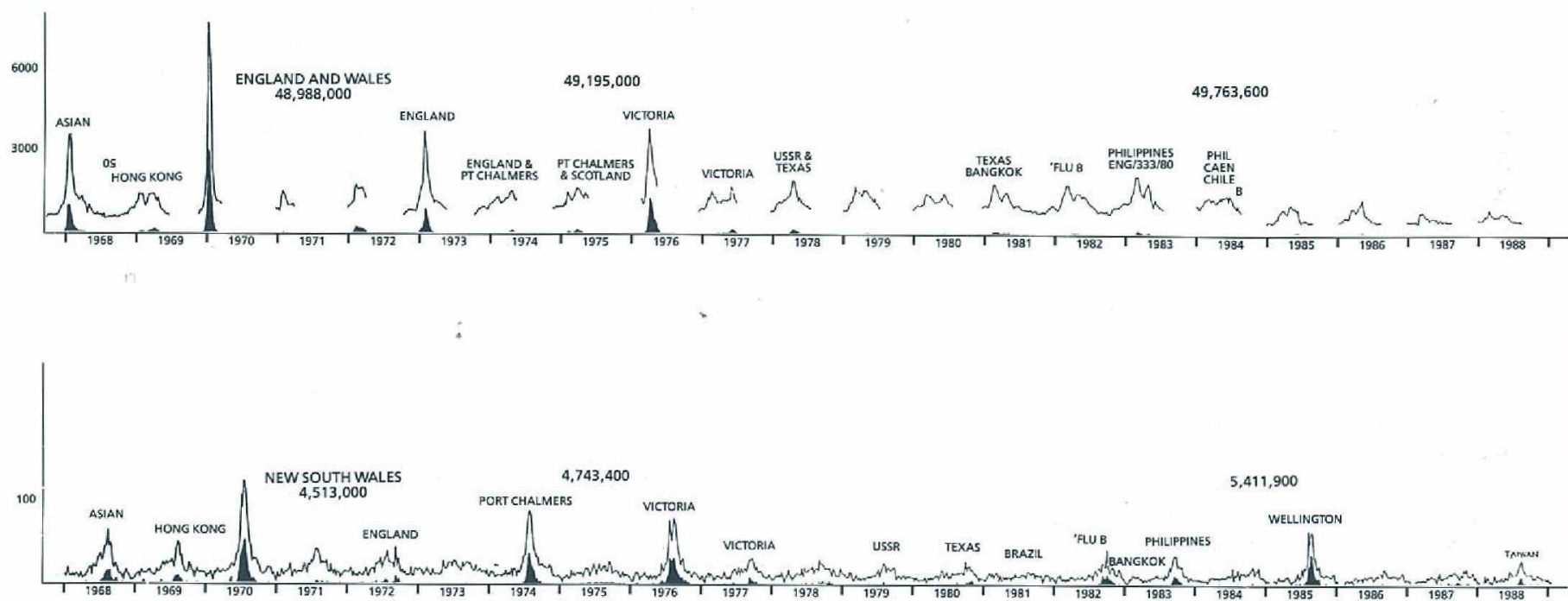

1. In each diagram the lower blacked-in graphs represent deaths attributed to Influenza (on death certificates), and the upper fine line graphs represent deaths from Influenza AND Pneumonia combined.

\section{- Continued from page 13}

\section{EDITORIAL NOTE:}

Despite the availability of an effective vaccine against influenza, the disease remains a major public health problem. It has the ability to paralyse essential services, schools and business and to cause substantial costs. Extrapolations from American data ${ }^{4}$ suggest that direct costs related to influenza for New South Wales in 1990 may be as high as $\$ 25,000,000$, with total costs 6-15 times this amount.

The present shortfall of influenza vaccine over demand has generated anxieties among many elderly people and others who consider that they have been denied the protection of immunisation. An alternative is to treat these patients with Amantadine, which provides some protection against influenza $\mathrm{A}$ - both prophylactically and therapeutically.5. Amantadine is marketed in Australia as Antadine ${ }^{\mathrm{R}}$ and Symmetrel ${ }^{\mathrm{R}}$. Reye's syndrome has been associated with influenza epidemics. Rates of 30.8 to 57.8 per million children infected with influenza have occurred in the United States - primarily in school aged children. With influenza attack rates of $25-40 \%^{7}$, NSW hospitals can expect to see 10-30 cases of Reye's syndrome this year.

Administration of aspirin may be implicated in the pathogenesis of Reye's syndrome. A causal relationship with aspirin may never be proven, but with adequate alternative treatment of pyrexia with paracetamol, there is no indication for the use of aspirin for temperature reduction in children or adolescents.
The Department of Health, NSW supports the National Health and Medical Research Council recommendations for immunisation against influenza, advising immunisations for:

(i) people of all ages with chronic debilitating disease, especially those with chronic cardiac, pulmonary, renal and metabolic disorders;

(ii) people over the age of 65 ;

(iii) people receiving immunosuppressive therapy; and (iv) people engaged in medical and health services, and essential public utilities.

PW Gill MB BS FRACGP (General Practitioner, Epping, NSW), $M H$ Levy $M B$ BS (Medical Epidemiologist, Epidemiology and Health Services Evaluation Branch, Department of Health, NSW), AL Cunningham MB BS MD FRACP (Virologist, WHO Collaborating Centre for Influenza Virology, Westmead Hospital).

1. Watson J. Public Health Laboratory Service, UK. Personal communication.

2. Communicable Diseases Intelligence. Bulletin 90/10. 21 May 1990. Canberra.

3. Gill PW, Cunningham AL, Murphy AM. Should healthy children be vaccinated against influenza? Lancet 1987;i:1440-1.

4. Prevention and Control of Influenza - Recommendations of the immunisation practices advisory committee. MMWR 1990;39:S1-15. 5. Douglas GR. Prophylaxis and treatment of influenza. N Eng J Med 1990;322:443-450.

6. Georges P. (ed) Report of the Committee on Infectious Diseases.

21st edition. American Academy of Pediatrics, Illinios, 1988.

7. Benenson AS (ed). Control of Communicable Diseases in Man. 14th edition. American Public Health Association. Washington DC. 1985. 